\title{
The genome sequence of the spectacle, Abrostola tripartita
}

\section{Hufnagel, 1766 [version 1; peer review: 3 approved]}

Douglas Boyes ${ }^{1+}$, Liam M. Crowley (D2), Peter W.H. Holland (iD2, University of Oxford and Wytham Woods Genome Acquisition Lab, Darwin Tree of Life Barcoding collective, Wellcome Sanger Institute Tree of Life programme, Wellcome Sanger Institute Scientific Operations: DNA Pipelines collective, Tree of Life Core Informatics collective, Darwin Tree of Life Consortium

${ }^{1}$ UK Centre for Ecology \& Hydrology, Wallingford, UK

${ }^{2}$ Department of Zoology, University of Oxford, Oxford, UK

+ Deceased author

V1 First published: $06 \operatorname{Dec} 2021,6: 330$

https://doi.org/10.12688/wellcomeopenres.17355.1

Latest published: 06 Dec 2021, 6:330

https://doi.org/10.12688/wellcomeopenres.17355.1

\section{Abstract}

We present a genome assembly from an individual male Abrostola tripartita (the spectacle; Arthropoda; Insecta; Lepidoptera; Noctuidae). The genome sequence is 381 megabases in span. The majority of the assembly (99.99\%) is scaffolded into 31 chromosomal pseudomolecules, with the $Z$ sex chromosome assembled.

Keywords

Abrostola tripartita, the spectacle, genome sequence, chromosomal, Lepidoptera

This article is included in the Tree of Life gateway.

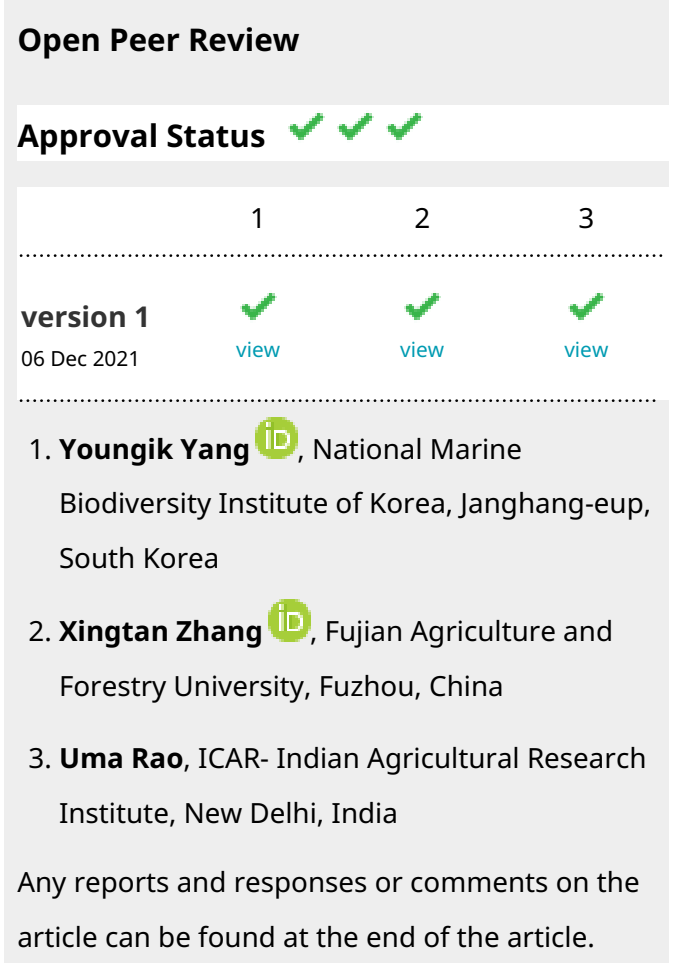


Corresponding author: Darwin Tree of Life Consortium (mark.blaxter@sanger.ac.uk)

Author roles: Boyes D: Investigation, Resources; Crowley LM: Writing - Original Draft Preparation, Writing - Review \& Editing; Holland PWH: Supervision, Writing - Original Draft Preparation, Writing - Review \& Editing;

Competing interests: No competing interests were disclosed.

Grant information: This work was supported by the Wellcome Trust through core funding to the Wellcome Sanger Institute (206194) and the Darwin Tree of Life Discretionary Award (218328).

The funders had no role in study design, data collection and analysis, decision to publish, or preparation of the manuscript.

Copyright: (c) 2021 Boyes D et al. This is an open access article distributed under the terms of the Creative Commons Attribution License, which permits unrestricted use, distribution, and reproduction in any medium, provided the original work is properly cited.

How to cite this article: Boyes D, Crowley LM, Holland PWH et al. The genome sequence of the spectacle, Abrostola tripartita Hufnagel, 1766 [version 1; peer review: 3 approved] Wellcome Open Research 2021, 6:330

https://doi.org/10.12688/wellcomeopenres.17355.1

First published: 06 Dec 2021, 6:330 https://doi.org/10.12688/wellcomeopenres.17355.1 


\section{Species taxonomy}

Eukaryota; Metazoa; Ecdysozoa; Arthropoda; Hexapoda; Insecta; Pterygota; Neoptera; Endopterygota; Lepidoptera; Glossata; Ditrysia; Noctuoidea; Noctuidae; Plusiinae; Abrostola; Abrostola tripartita Hufnagel, 1766 (NCBI:txid938171).

\section{Background}

Abrostola tripartita (the spectacle) is a grey and white noctuid moth recorded from across the Palaearctic region. It is found commonly across the UK where it has increased significantly in abundance in recent decades (Sterling \& Henwood, 2020). The common name derives from two rings of grey hairs on the thorax with the appearance of a pair of goggles, visible when the moth is viewed from anterior; these hairs are on the thorax and are not associated with the head or eyes. The larvae feed on nettle (Urtica dioica) and may be found across any habitat where the food plant is in abundance. Adults often feed at flowers including red valerian (Centranthus ruber) and sage (Salvia). In the UK the adult flight period occurs as a single generation in the north (May to July) and as two generations in the south (May-July and July-September) (Waring et al., 2003). Overwintering occurs as a pupa among plant litter on the ground or under bark.

\section{Genome sequence report}

The genome was sequenced from one male $A$. tripartita (Figure 1) collected from Wytham Woods, Oxfordshire (biological vice-county: Berkshire), UK (latitude 51.769, longitude -1.339). A total of 54-fold coverage in Pacific Biosciences single-molecule long reads and 102-fold coverage in 10X Genomics read clouds were generated. Primary assembly contigs were scaffolded with chromosome conformation Hi-C data. Manual assembly curation corrected 30 missing/misjoins and removed 3 haplotypic duplications, reducing the assembly size by $0.44 \%$ and the scaffold number by $20.00 \%$.

The final assembly has a total length of $381 \mathrm{Mb}$ in 32 sequence scaffolds with a scaffold N50 of $13.6 \mathrm{Mb}$ (Table 1). The majority of the assembly sequence $(99.99 \%)$ was assigned to 31 chromosomal-level scaffolds, representing 30 autosomes (numbered by sequence length), and the $\mathrm{Z}$ sex chromosome (Figure 2-Figure 5; Table 2). The assembly has a BUSCO v5.1.2 (Manni et al., 2021) completeness of $99.0 \%$ (single $98.8 \%$, duplicated $0.2 \%$ ) using the lepidoptera_odb10 reference set. While not fully phased, the assembly deposited is of one haplotype. Contigs corresponding to the second haplotype have also been deposited.

\section{Methods}

A single male A. tripartita (ilAbrTrip1) was collected from Wytham Woods, Oxfordshire (biological vice-county: Berkshire), UK (latitude 51.769, longitude -1.339) by Douglas Boyes, UKCEH, using a light trap. The sample was identified by the same individual, and preserved on dry ice.

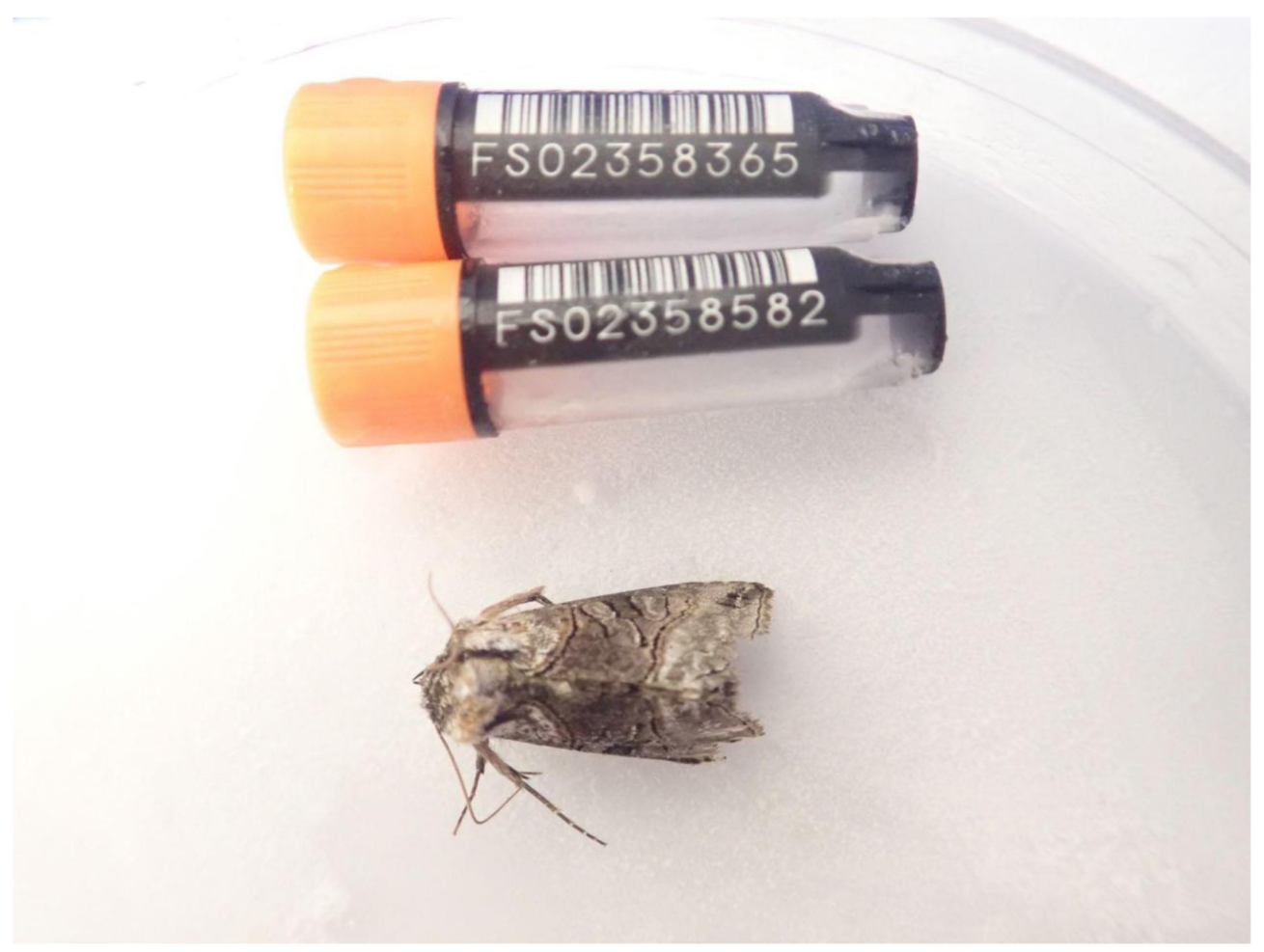

Figure 1. Image of the ilAbrTrip1 specimen taken prior to preservation and processing. Specimen shown next to FluidX storage tube, $43.9 \mathrm{~mm}$ in length. 
Table 1. Genome data for Abrostola tripartita, ilAbrTrip1.1.

\begin{tabular}{|l|l|}
\hline Project accession data & \\
\hline Assembly identifier & ilAbrTrip1.1 \\
\hline Species & Abrostola tripartita \\
\hline Specimen & ilAbrTrip1 \\
\hline NCBI taxonomy ID & NCBI:txid938171 \\
\hline BioProject & PRJEB43740 \\
\hline BioSample ID & SAMEA7520667 \\
\hline Isolate information & Male, thorax (genome assembly), head (Hi-C), abdomen (RNA-Seq) \\
\hline
\end{tabular}

Raw data accessions

PacificBiosciences SEQUEL II

ERR6412364

10X Genomics Illumina

ERR6054553-ERR6054556

Hi-C Illumina

ERR6054552

Illumina polyA RNA-Seq

ERR6054557

\section{Genome assembly}

Assembly accession GCA_905340225.1

Accession of alternate haplotype GCA_905340255.1

Span (Mb) 381

Number of contigs 34

Contig N50 length (Mb) $\quad 13.6$

\begin{tabular}{l|l} 
Number of scaffolds & 32
\end{tabular}

Scaffold N50 length (Mb) $\quad 13.6$

\begin{tabular}{l|l} 
Longest scaffold $(\mathrm{Mb})$ & 16.0
\end{tabular}

BUSCO* genome score C:99.0\%[S:98.8\%,D:0.2\%],F:0.3\%,M:0.7\%,n:5286

*BUSCO scores based on the lepidoptera_odb10 BUSCO set using v5.1.2. C= complete [S= single copy, $\mathrm{D}=$ duplicated], $\mathrm{F}=$ fragmented, $\mathrm{M}=$ missing, $\mathrm{n}=$ number of orthologues in comparison. A full set of BUSCO scores is available at https:// blobtoolkit.genomehubs.org/view/ilAbrTrip1.1/dataset/CAJPHX01/busco.

DNA was extracted at the Tree of Life laboratory, Wellcome Sanger Institute. The ilAbrTrip1 sample was weighed and dissected on dry ice with tissue set aside for Hi-C and RNA sequencing. Thorax tissue was cryogenically disrupted to a fine powder using a Covaris cryoPREP Automated Dry Pulveriser, receiving multiple impacts. Fragment size analysis of 0.01-0.5 ng of DNA was then performed using an Agilent FemtoPulse. High molecular weight (HMW) DNA was extracted using the Qiagen MagAttract HMW DNA extraction kit. Low molecular weight DNA was removed from a 200-ng aliquot of extracted DNA using $0.8 \mathrm{X}$ AMpure XP purification kit prior to $10 \mathrm{X}$ Chromium sequencing; a minimum of $50 \mathrm{ng}$ DNA was submitted for $10 \mathrm{X}$ sequencing. HMW DNA was sheared into an average fragment size between $12-20 \mathrm{~kb}$ in a Megaruptor 3 system with speed setting 30. Sheared DNA was purified by solid-phase reversible immobilisation using AMPure PB beads with a $1.8 \mathrm{X}$ ratio of beads to sample to remove the shorter fragments and concentrate the DNA sample. The concentration of the sheared and purified DNA was assessed using a Nanodrop spectrophotometer and Qubit Fluorometer and Qubit dsDNA High Sensitivity Assay kit. Fragment size distribution was evaluated by running the sample on the FemtoPulse system.

RNA was extracted from abdomen tissue in the Tree of Life Laboratory at the WSI using TRIzol (Invitrogen), according to the manufacturer's instructions. RNA was then eluted in $50 \mu \mathrm{l}$ RNAse-free water and its concentration assessed using a Nanodrop spectrophotometer and Qubit Fluorometer using the Qubit RNA Broad-Range (BR) Assay kit. Analysis of the 


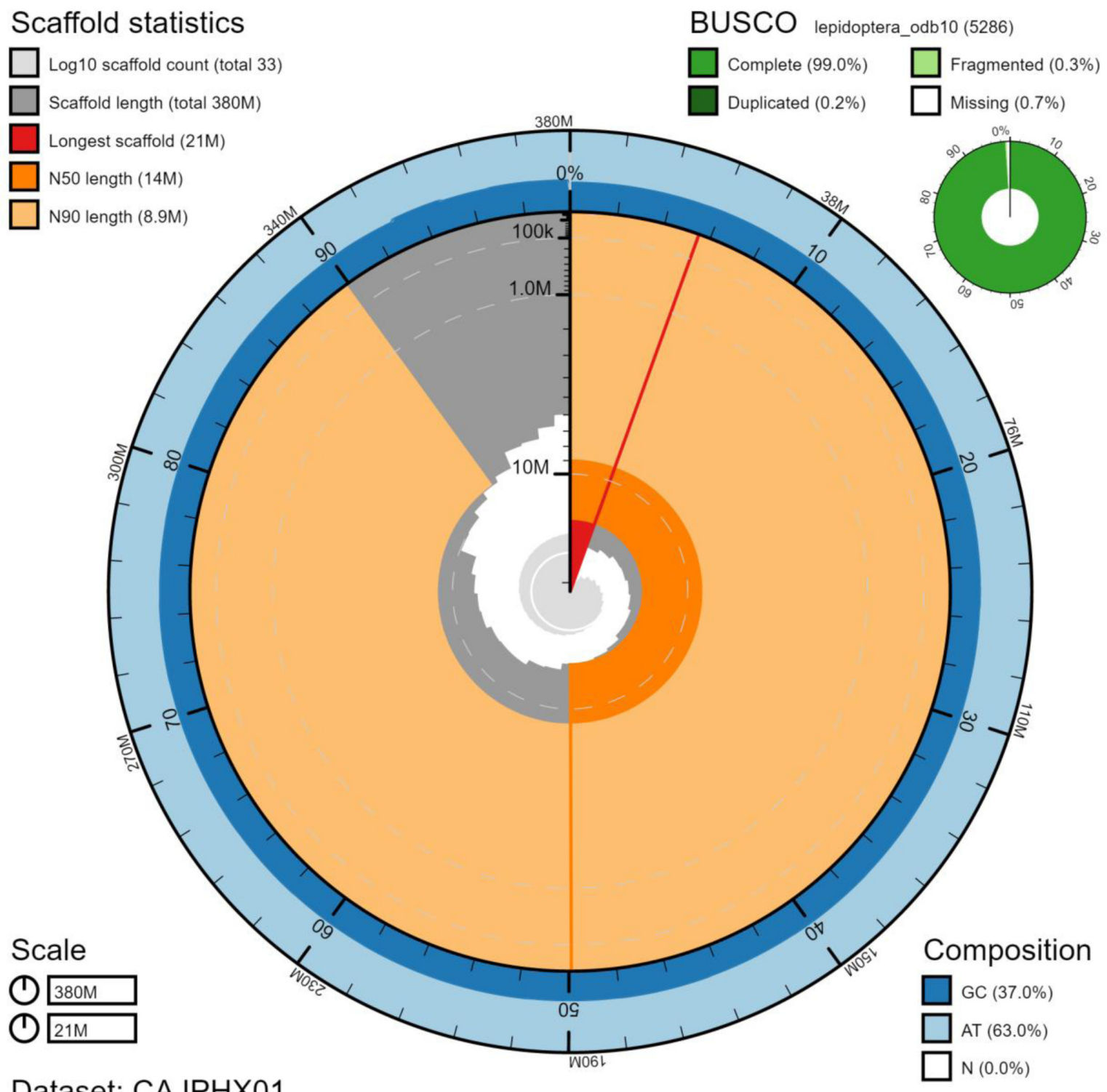

Figure 2. Genome assembly of Abrostola tripartita, ilAbrTrip1.1: metrics. The BlobToolKit Snailplot shows N50 metrics and BUSCO gene completeness. The main plot is divided into 1,000 size-ordered bins around the circumference with each bin representing $0.1 \%$ of the $381,057,457$ bp assembly. The distribution of chromosome lengths is shown in dark grey with the plot radius scaled to the longest chromosome present in the assembly (20,963,991 bp, shown in red). Orange and pale-orange arcs show the N50 and N90 chromosome lengths $(13,645,312$ and 8,916,110 bp), respectively. The pale grey spiral shows the cumulative chromosome count on a log scale with white scale lines showing successive orders of magnitude. The blue and pale-blue area around the outside of the plot shows the distribution of GC, AT and N percentages in the same bins as the inner plot. A summary of complete, fragmented, duplicated and missing BUSCO genes in the lepidoptera_odb10 set is shown in the top right. An interactive version of this figure is available at https://blobtoolkit.genomehubs. org/view/ilAbrTrip1.1/dataset/CAJPHX01/snail.

integrity of the RNA was done using Agilent RNA 6000 Pico Kit and Eukaryotic Total RNA assay.

\section{Sequencing}

Pacific Biosciences HiFi circular consensus and 10X Genomics Chromium read cloud sequencing libraries were constructed according to the manufacturers' instructions. Poly(A) RNA-Seq libraries were constructed using the NEB Ultra II RNA Library Prep kit. Sequencing was performed by the Scientific Operations core at the Wellcome Sanger Institute on Pacific Biosciences SEQUEL II (HiFi), Illumina HiSeq $\mathrm{X}$ (10X) and Illumina HiSeq 4000 (RNA-Seq) instruments. $\mathrm{Hi}-\mathrm{C}$ data were generated from head tissue using the Arima $\mathrm{Hi}-\mathrm{C}+$ kit and sequenced on HiSeq X. 


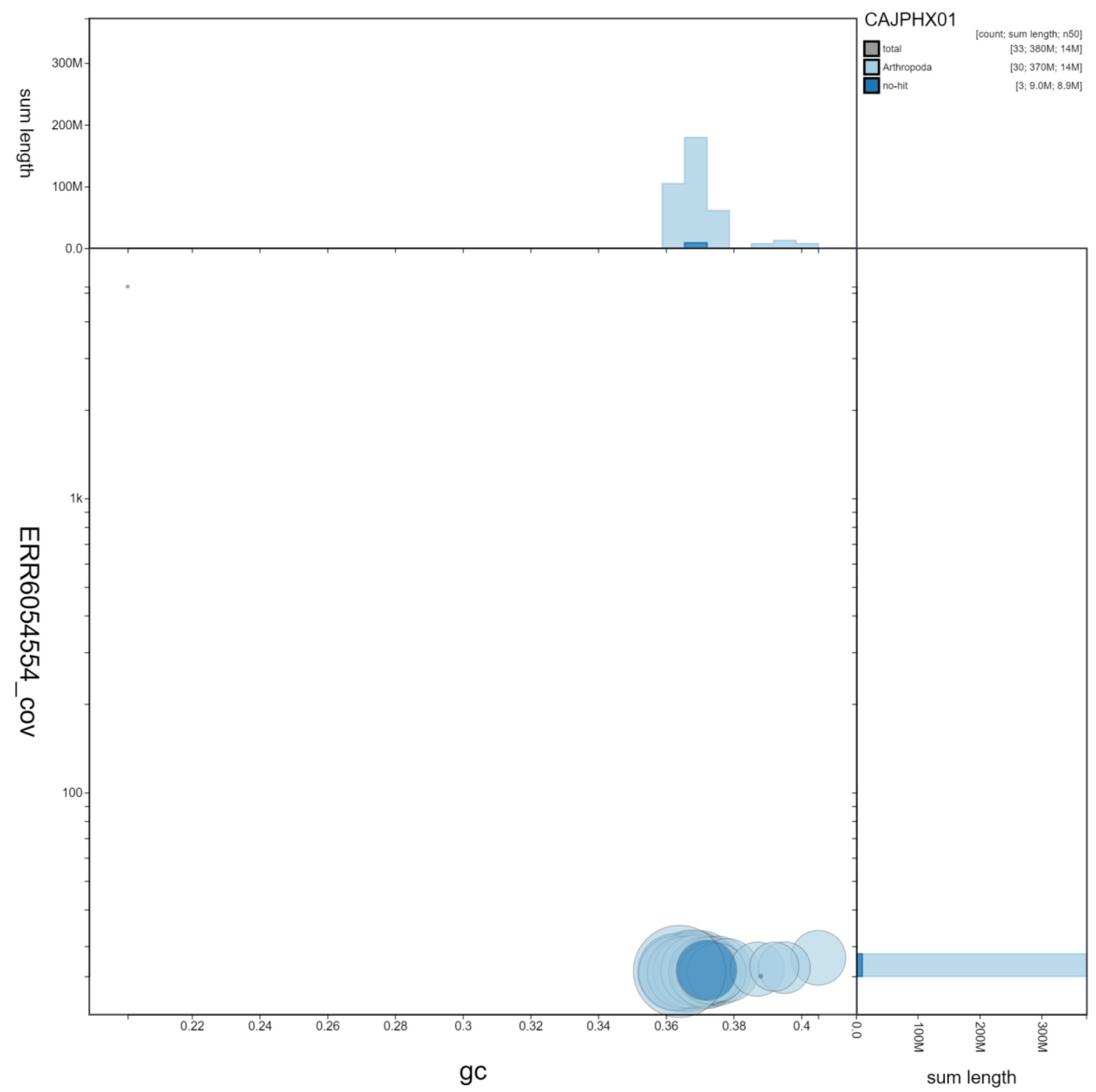

Figure 3. Genome assembly of Abrostola tripartita, ilAbrTrip1.1: GC coverage. BlobToolKit GC-coverage plot. Scaffolds are coloured by phylum. Circles are sized in proportion to scaffold length Histograms show the distribution of scaffold length sum along each axis. An interactive version of this figure is available at https://blobtoolkit.genomehubs.org/view/ilAbrTrip1.1/dataset/CAJPHX01/blob.

\section{Genome assembly}

Assembly was carried out with Hifiasm (Cheng et al., 2021); haplotypic duplication was identified and removed with purge_dups (Guan et al., 2020). One round of polishing was performed by aligning $10 \mathrm{X}$ Genomics read data to the assembly with longranger align, calling variants with freebayes
(Garrison \& Marth, 2012). The assembly was then scaffolded with Hi-C data (Rao et al., 2014) using SALSA2 (Ghurye et al., 2019). The assembly was checked for contamination and corrected using the gEVAL system (Chow et al., 2016) as described previously (Howe et al., 2021). Manual curation (Howe et al., 2021) was performed using gEVAL, HiGlass 


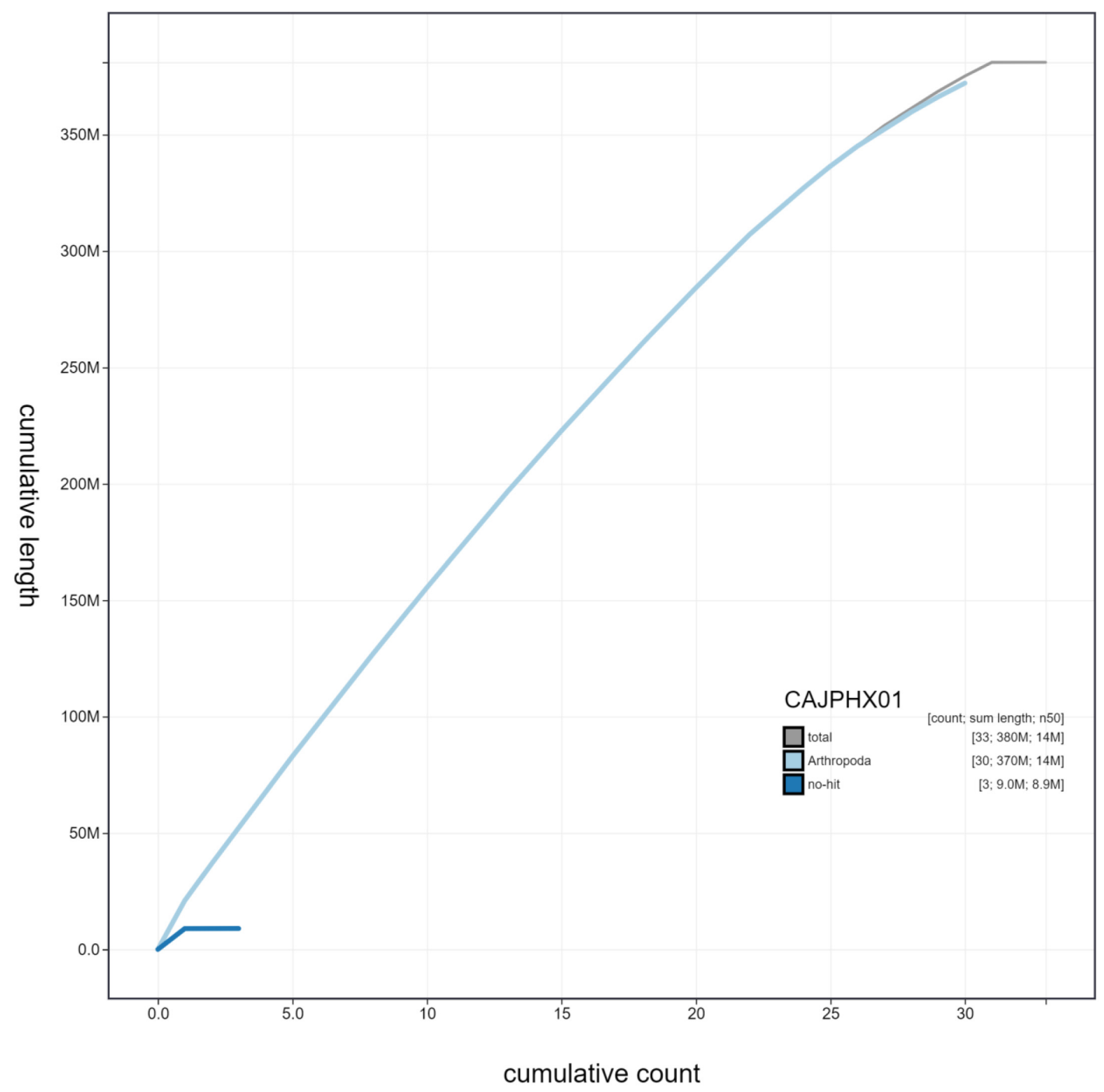

Figure 4. Genome assembly of Abrostola tripartita, ilAbrTrip1.1: cumulative sequence. BlobToolKit cumulative sequence plot. The grey line shows cumulative length for all scaffolds. Coloured lines show cumulative lengths of scaffolds assigned to each phylum using the buscogenes taxrule. An interactive version of this figure is available at https://blobtoolkit.genomehubs.org/view/ilAbrTrip1.1/dataset/ CAJPHX01/cumulative.

(Kerpedjiev et al., 2018) and Pretext. The mitochondrial genome was assembled using MitoHiFi (Uliano-Silva et al., 2021). The genome was analysed and BUSCO scores generated within the BlobToolKit environment (Challis et al., 2020). Table 3 contains a list of all software tool versions used, where appropriate.

\section{Ethics/compliance issues}

The materials that have contributed to this genome note have been supplied by a Darwin Tree of Life Partner. The submission of materials by a Darwin Tree of Life Partner is subject to the Darwin Tree of Life Project Sampling Code of Practice. By agreeing with and signing up to the Sampling Code of Practice, 


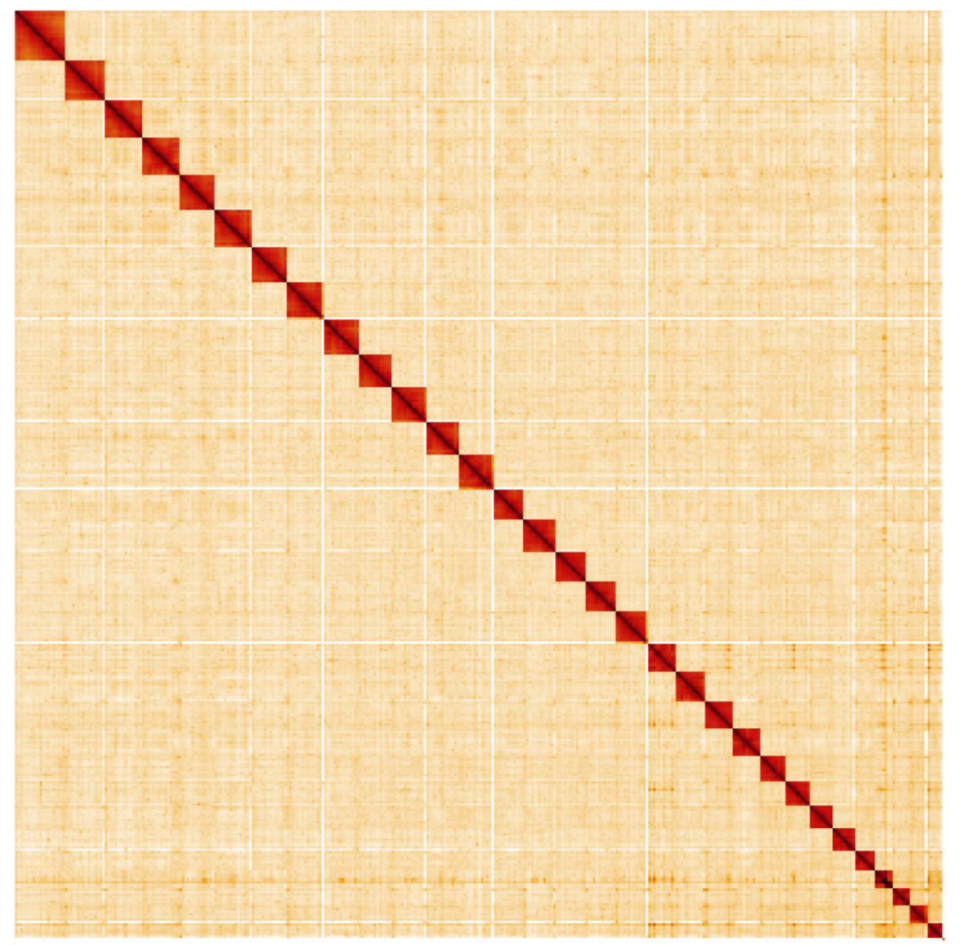

Figure 5. Genome assembly of Abrostola tripartita, ilAbrTrip1.1: Hi-C contact map. Hi-C contact map of the ilAbrTrip1.1 assembly, visualised in HiGlass.

Table 2. Chromosomal pseudomolecules in the genome assembly of Abrostola tripartita, ilAbrTrip1.1.

\begin{tabular}{|c|c|c|c|}
\hline INSDC accession & Chromosome & Size (Mb) & GC\% \\
\hline HG996487.1 & 1 & 15.98 & 36.7 \\
\hline HG996488.1 & 2 & 15.59 & 36.8 \\
\hline HG996489.1 & 3 & 15.22 & 37 \\
\hline HG996490.1 & 4 & 15.13 & 36.7 \\
\hline HG996491.1 & 5 & 14.91 & 36.8 \\
\hline HG996492.1 & 6 & 14.74 & 36.3 \\
\hline HG996493.1 & 7 & 14.47 & 36.5 \\
\hline HG996494.1 & 8 & 14.45 & 36.3 \\
\hline HG996495.1 & 9 & 14.04 & 36.6 \\
\hline HG996496.1 & 10 & 13.84 & 36.3 \\
\hline HG996497.1 & 11 & 13.71 & 36.7 \\
\hline HG996498.1 & 12 & 13.65 & 36.3 \\
\hline HG996499.1 & 13 & 13.10 & 36.7 \\
\hline HG996500.1 & 14 & 12.99 & 36.7 \\
\hline HG996501.1 & 15 & 12.52 & 36.5 \\
\hline
\end{tabular}

\begin{tabular}{|c|c|c|c|}
\hline INSDC accession & Chromosome & Size (Mb) & GC\% \\
\hline HG996502.1 & 16 & 12.52 & 36.9 \\
\hline HG996503.1 & 17 & 12.43 & 37.2 \\
\hline HG996504.1 & 18 & 12.03 & 37.2 \\
\hline HG996505.1 & 19 & 11.91 & 37.5 \\
\hline HG996506.1 & 20 & 11.64 & 37.1 \\
\hline HG996507.1 & 21 & 11.29 & 37.3 \\
\hline HG996508.1 & 22 & 10.01 & 37.4 \\
\hline HG996509.1 & 23 & 9.94 & 37.8 \\
\hline HG996510.1 & 24 & 9.43 & 37.3 \\
\hline HG996511.1 & 25 & 8.92 & 37.2 \\
\hline HG996512.1 & 26 & 8.52 & 37.5 \\
\hline HG996513.1 & 27 & 7.37 & 40.5 \\
\hline HG996514.1 & 28 & 7.24 & 38.7 \\
\hline HG996515.1 & 29 & 6.58 & 39.5 \\
\hline HG996516.1 & 30 & 5.91 & 39.2 \\
\hline HG996486.1 & $Z$ & 20.96 & 36.4 \\
\hline HG996517.1 & MT & 0.02 & 19.9 \\
\hline & Unplaced & 0.03 & 38.5 \\
\hline- & 25 & \\
\hline
\end{tabular}




\begin{tabular}{|c|c|c|}
\hline Software tool & Version & Source \\
\hline Hifiasm & 0.142 & Cheng et al., 2021 \\
\hline purge_dups & 1.2 .3 & Guan et al., 2020 \\
\hline SALSA2 & 2.2 & Ghurye et al., 2019 \\
\hline longranger align & 2.2 .2 & $\begin{array}{l}\text { https://support.10xgenomics.com/ } \\
\text { genome-exome/software/pipelines/latest/ } \\
\text { advanced/other-pipelines }\end{array}$ \\
\hline freebayes & 1.3.1-17-gaa2ace8 & Garrison \& Marth, 2012 \\
\hline MitoHiFi & 1.0 & Uliano-Silva et al., 2021 \\
\hline gEVAL & N/A & Chow et al., 2016 \\
\hline HiGlass & 1.11 .6 & Kerpedjiev et al., 2018 \\
\hline PretextView & $0.1 . x$ & https://github.com/wtsi-hpag/PretextView \\
\hline BlobToolKit & 2.6 .2 & Challis et al., 2020 \\
\hline
\end{tabular}

the Darwin Tree of Life Partner agrees they will meet the legal and ethical requirements and standards set out within this document in respect of all samples acquired for, and supplied to, the Darwin Tree of Life Project. Each transfer of samples is further undertaken according to a Research Collaboration Agreement or Material Transfer Agreement entered into by the Darwin Tree of Life Partner, Genome Research Limited (operating as the Wellcome Sanger Institute), and in some circumstances other Darwin Tree of Life collaborators.

\section{Data availability}

European Nucleotide Archive: Abrostola tripartita (the spectacle). Accession number PRJEB43740; https://identifiers.org/ena.embl/ PRJEB43740.

The genome sequence is released openly for reuse. The A. tripartita genome sequencing initiative is part of the Darwin Tree of Life (DToL) project. All raw sequence data and the assembly have been deposited in INSDC databases. The genome will be annotated using the RNA-Seq data and presented through the Ensembl pipeline at the European Bioinformatics Institute. Raw data and assembly accession identifiers are reported in Table 1.

\section{Author information}

Members of the University of Oxford and Wytham Woods Genome Acquisition Lab are listed here: https://doi.org/10.5281/ zenodo.4789929.

Members of the Darwin Tree of Life Barcoding collective are listed here: https://doi.org/10.5281/zenodo.4893704.

Members of the Wellcome Sanger Institute Tree of Life programme collective are listed here: https://doi.org/10.5281/zenodo. 5377053.

Members of Wellcome Sanger Institute Scientific Operations: DNA Pipelines collective are listed here: https://doi.org/10.5281/ zenodo.4790456.

Members of the Tree of Life Core Informatics collective are listed here: https://doi.org/10.5281/zenodo.5013542.

Members of the Darwin Tree of Life Consortium are listed here: https://doi.org/10.5281/zenodo.4783559.
Challis R, Richards E, Rajan J, et al.: BlobToolKit - Interactive Quality Assessment of Genome Assemblies. G3 (Bethesda). 2020; 10(4): 1361-74. PubMed Abstract | Publisher Full Text | Free Full Text

Cheng H, Concepcion GT, Feng X, et al.: Haplotype-Resolved de Novo Assembly Using Phased Assembly Graphs with Hifiasm. Nat Methods. 2021;
18(2): 170-75.

PubMed Abstract | Publisher Full Text | Free Full Text Chow W, Brugger K, Caccamo M, et al.: gEVAL - a web-based browser for evaluating genome assemblies. Bioinformatics. 2016; 32(16): 2508-10. PubMed Abstract | Publisher Full Text | Free Full Text 
Garrison E, Marth G: Haplotype-Based Variant Detection from Short-Read Sequencing. arXiv: 1207.3907. 2012

Reference Source

Ghurye J, Rhie A, Walenz BP, et al.: Integrating Hi-C Links with Assembly Graphs for Chromosome-Scale Assembly. PLoS Comput Biol. 2019; 15(8): e1007273.

PubMed Abstract | Publisher Full Text | Free Full Text

Guan D, McCarthy SA, Wood J, et al.: Identifying and Removing Haplotypic Duplication in Primary Genome Assemblies. Bioinformatics. 2020; 36(9): 2896-98.

PubMed Abstract | Publisher Full Text | Free Full Text

Howe K, Chow W, Collins J, et al.: Significantly Improving the Quality of Genome Assemblies through Curation. Gigascience. 2021; 10(1): giaa153. PubMed Abstract | Publisher Full Text | Free Full Text

Kerpedjiev P, Abdennur N, Lekschas F, et al:: HiGlass: Web-Based Visual Exploration and Analysis of Genome Interaction Maps. Genome Biol. 2018; 19(1): 125

PubMed Abstract | Publisher Full Text | Free Full Text
Manni M, Berkeley MR, Seppey M, et al.: BUSCO Update: Novel and Streamlined Workflows along with Broader and Deeper Phylogenetic Coverage for Scoring of Eukaryotic, Prokaryotic, and Viral Genomes. $\mathrm{Mol}$ Biol Evol. 2021; 38(10): 4647-54.

PubMed Abstract | Publisher Full Text | Free Full Text

Rao SSP, Huntley MH, Durand NC, et al.: A 3D Map of the Human Genome at Kilobase Resolution Reveals Principles of Chromatin Looping. Cell. 2014 159(7): 1665-80.

PubMed Abstract | Publisher Full Text | Free Full Text

Sterling P, Henwood B: Field Guide to the Caterpillars of Great Britain and Ireland. Bloomsbury Publishing. 2020.

Reference Source

Uliano-Silva M, Nunes JGF, Krasheninnikova K, et al.: marcelauliano/MitoHiFi: mitohifi_v2.0. 2021.

Publisher Full Text

Waring $P$, Townsend $M$, Lewington R: Field Guide to the Moths of Great Britain and Ireland. British Wildlife Publishing, Hampshire. 2003. Reference Source 


\section{Open Peer Review}

\section{Current Peer Review Status:}

\section{Version 1}

Reviewer Report 25 January 2022

https://doi.org/10.21956/wellcomeopenres.19185.r47467

(C) 2022 Rao U. This is an open access peer review report distributed under the terms of the Creative Commons Attribution License, which permits unrestricted use, distribution, and reproduction in any medium, provided the original work is properly cited.

\section{Uma Rao}

Division of Nematology, ICAR- Indian Agricultural Research Institute, New Delhi, Delhi, India

The paper is an announcement and is written succinctly. All methods are adequate and results are sufficient to explain the findings. It is recommend for indexing with one minor edit:

There is one mismatch between data in text and figure. Table 1 and text report the longest scaffold as $16.0 \mathrm{Mb}$, whereas figure 2 shows it as $21 \mathrm{Mb}$, which should be corrected.

Is the rationale for creating the dataset(s) clearly described?

Yes

Are the protocols appropriate and is the work technically sound?

Yes

Are sufficient details of methods and materials provided to allow replication by others? Yes

Are the datasets clearly presented in a useable and accessible format? Yes

Competing Interests: No competing interests were disclosed.

Reviewer Expertise: Plant nematode interactions, genomics, transgenics

I confirm that I have read this submission and believe that I have an appropriate level of expertise to confirm that it is of an acceptable scientific standard.

Reviewer Report 11 January 2022

https://doi.org/10.21956/wellcomeopenres.19185.r47462 
(c) 2022 Zhang X. This is an open access peer review report distributed under the terms of the Creative Commons Attribution License, which permits unrestricted use, distribution, and reproduction in any medium, provided the original work is properly cited.

\section{Xingtan Zhang}

State Key Laboratory of Ecological Pest Control for Fujian and Taiwan Crops, Institute of Applied Ecology, College of Plant Protection, Fujian Agriculture and Forestry University, Fuzhou, China

The authors provide a high-quality chromosome-scale genome assembly of Abrostola tripartita. I believe these resources will be useful for the community. I only have two comments on this manuscript:

1. The authors used Arima $\mathrm{Hi}-\mathrm{C}+\mathrm{kit}$ to construct the $\mathrm{Hi}-\mathrm{C}$ but provide limited methods. What is the difference between Arima $\mathrm{Hi}-\mathrm{C}$ and other related $\mathrm{Hi}-\mathrm{C}$ methods? Similarly, the SALSA scaffolder was used without details. More information should be added.

2. The quality of genome assembly was only accessed using BUSCO and heatmap. I recommend adding more analysis to valid the genome assembly. For instance, the authors could map sequencing reads to their assembly and check the distribution of coverage along each chromosome.

Is the rationale for creating the dataset(s) clearly described?

Yes

Are the protocols appropriate and is the work technically sound? Yes

Are sufficient details of methods and materials provided to allow replication by others? Partly

Are the datasets clearly presented in a useable and accessible format?

Yes

Competing Interests: No competing interests were disclosed.

Reviewer Expertise: Genomics

I confirm that I have read this submission and believe that I have an appropriate level of expertise to confirm that it is of an acceptable scientific standard.

Reviewer Report 05 January 2022

https://doi.org/10.21956/wellcomeopenres.19185.r47465

(C) 2022 Yang Y. This is an open access peer review report distributed under the terms of the Creative Commons Attribution License, which permits unrestricted use, distribution, and reproduction in any medium, provided the original work is properly cited. 


\section{Youngik Yang}

National Marine Biodiversity Institute of Korea, Janghang-eup, South Korea

The data note by Boyes et al. presented a high-quality genome assembly of Abrostola tripartita, a grey and white noctuid moth. They applied state-of-the-art sequencing technologies and bioinformatics methods. The resulting assembly contained 32 pseudo-chromosomes and BUSCO was $99 \%$. I believe that this high-quality chromosome-level assembly will be a valuable resource for further studies of this species as well as comparative studies with other species.

I have some recommendations for improving the article.

1. Specimen image would be better to be shown next to the ruler not the FluidX storage.

2. Please, provide the exact number of chromosomes and references if available.

3. MitoHiFi requires a close-related species for input. Please, provide what it was.

4. Figure 2 and 3 are not very informative in my opinion. The information in the figures can be combined in table 2.

Is the rationale for creating the dataset(s) clearly described?

Yes

Are the protocols appropriate and is the work technically sound?

Yes

Are sufficient details of methods and materials provided to allow replication by others? Yes

Are the datasets clearly presented in a useable and accessible format?

Yes

Competing Interests: No competing interests were disclosed.

Reviewer Expertise: Bioinformatics, Genomics

I confirm that I have read this submission and believe that I have an appropriate level of expertise to confirm that it is of an acceptable scientific standard. 